\title{
Probing the Cytotoxicity Of Semiconductor Quantum Dots
}

\author{
Austin M. Derfus ${ }^{1}$, Warren C.W. Chan ${ }^{1,2}$, and Sangeeta N. Bhatia ${ }^{1, *}$ \\ ${ }^{1}$ Dept of Bioengineering, University of California at San Diego, La Jolla, California, USA
}

\begin{abstract}
With their bright, photostable fluorescence, semiconductor quantum dots show promise as alternatives to organic dyes for biological labeling. Questions about their potential cytotoxicity, however, remain unanswered. While cytotoxicity of bulk cadmium selenide (CdSe) is well documented, a number of groups have suggested that CdSe QDs are cytocompatible, at least with some immortalized cell lines. Using primary hepatocytes as a liver model, we found that CdSecore QDs were indeed acutely toxic under certain conditions. Specifically, we found that the cytotoxicity of QDs was modulated by processing parameters during synthesis, exposure to ultraviolet light, and surface coatings. Our data further suggests that cytotoxicity correlates with the liberation of free $\mathrm{Cd}^{2+}$ ions due to deterioration of the CdSe lattice. When appropriately coated, CdSe-core QDs can be rendered non-toxic and used to track cell migration and reorganization in vitro. Our results inform design criteria for the use of QDs in vitro and especially in vivo where deterioration over time may occur.
\end{abstract}

\section{Graphical Abstract}

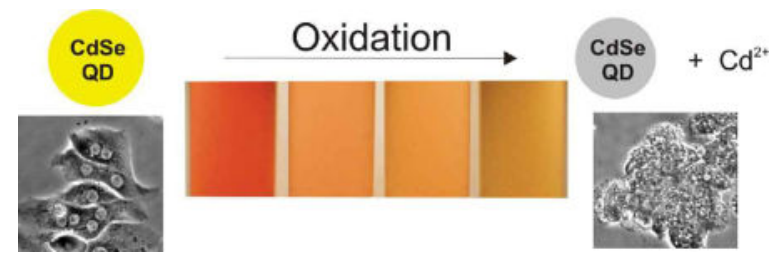

\section{Keywords}

quantum dots; nanocrystals; nanoparticles; toxicity; cadmium

Recently, nanomaterials have received enormous attention for their potential applications in biology and medicine. A key issue in evaluating the utility of these materials is assessing their potential toxicity - either due to their inherent chemical composition (e.g. heavy metals) or as a consequence of their nanoscale properties (e.g. inhalation of particulate carbon nanotubes ${ }^{1,2}$ ). CdSe-core quantum dots are an example of a nanomaterial that has

\footnotetext{
"Correspondence should be addressed to: Sangeeta N. Bhatia, MD, PhD, Departments of Bioengineering \& Medicine, UCSD- 9500 Gilman Dr. MC 0412, La Jolla, CA 92093, Tel: 858-822-3142, Fax: 858-822-4203, sbhatia@ ucsd.edu.

${ }^{2}$ Current Address: Institute of Biomaterials and Biomedical Engineering (IBBME), University of Toronto, Toronto, Ontario, CANADA

Supporting Information Available: Experimental Methods. This information is available free of charge via the Internet at http:// pubs.acs.org.
} 
been shown useful as an alternative to fluorescent dyes for use in biological imaging, due to their bright fluorescence, narrow emission, broad UV excitation and high photostability ${ }^{3-5}$. In addition to labeling of cellular structures in vitro ${ }^{3,6-10}$, several groups have demonstrated the use of QDs for fluorescence imaging in vivo ${ }^{11-13}$.

As the popularity of QD labeling soars, however, concerns are beginning to surface about the toxicity of these cadmium-containing nanoparticles. Several commentaries have recently posed the question of QD toxicity, but no solid evidence exists to provide a definitive answer ${ }^{14-16}$. There has been considerable concern that the inherently toxic elements of the QD core (e.g., cadmium, selenium) would render the nanoparticles toxic to both cell cultures and live animals. While previous in vitro labeling experiments have not shown significant toxicity, most have used short-term QD labeling and immortalized cell lines that are not highly sensitive to heavy metals ${ }^{6,8,9}$. Additionally, a relevant model for in vivo toxicity should be examined, as design criteria and constraints for preventing cytotoxicity in vivo are needed.

In vivo, the liver is the primary site of acute injury due to Cd exposure, and even low levels of cadmium ions $(100-400 \mu \mathrm{M})$ are known to reduce the viability of hepatocytes in vitro ${ }^{17}$. Furthermore, greater than $25 \%$ of the cadmium administered to rats accumulates in the liver ${ }^{18}$. Cadmium binding to sulfhydryl groups of critical mitochondrial proteins is thought to be the mechanism of hepatic injury ${ }^{19}$. Thiol group inactivation then leads to oxidative stress and mitochondrial dysfunction. Metallothionein, a protein found in the cytoplasm of hepatocytes, detoxifies cadmium by sequestering it into an inert complex. Yet, the small amounts of this protein normally present in animals is not sufficient when cells are exposed to high levels of $\mathrm{Cd}^{2+}$ ions. Hepatocytes isolated from rats and grown in vitro have metallothionein levels similar to those in the intact liver, and thus isolated cells serve as representative model for in vivo hepatotoxicity ${ }^{20}$. Since the liver is a major target of $\mathrm{Cd}$ injury, primary hepatocyte culture is an ideal model to investigate the cytotoxicity of QDs.

A variety of syntheses, storage, coating strategies have been proposed for CdSe QDs ${ }^{21-25}$. While long-term animal studies remains the gold standard for toxicology, examination of extreme conditions in appropriate in vitro models is a well-established approach to evaluate the toxicity of novel materials and is therefore the strategy pursued in this study. Our initial observations led us to conclude that process parameters and environmental conditions could dramatically affect the observed toxicity in a hepatocyte culture model. Furthermore, the cytotoxicity was affected by the degree and stability of the QD surface coating. We therefore set out to systematically examine the role of process parameters, surface coatings, and UV excitation on the rate of cytotoxicity as well as establish mechanistic framework to unify our findings and establish a benchmark for determining the biocompatibility of novel surface coatings.

\section{Cytotoxicity of CdSe Quantum Dots In Vitro}

In order to establish a model system for rigorous evaluation of cytotoxicity, primary hepatocytes were isolated from rats, cultured in monolayers and incubated with yellowemitting CdSe QDs $\left(\lambda_{\mathrm{em} \max }=582 \mathrm{~nm}\right.$ ) for 24 hours (Figure 1). QDs were prepared using a 
high-temperature organometallic procedure with the solvent tri-n-octylphosphine oxide (TOPO) in an inert atmosphere ${ }^{25}$, water-solubilized with mercaptoacetic acid (MAA) ${ }^{3}$, and delivered over a range of typical doses for both in vitro and in vivo experimentation (1.0, $0.25,0.0625 \mathrm{mg} / \mathrm{mL}$ ). We chose MAA because it is one of the smallest solubilization ligands and thus the least protective of the QD surface ${ }^{26}$. We perturbed the QD structure by exposing TOPO-capped QDs to air or illuminating with high-energy radiation and determined the influence of these parameters on cellular viability. Cytotoxicity was evaluated by MTT viability assay, a colorimetric measure of mitochondrial activity (Figure 1A), and morphologically with bright-field microscopy (Figures 1B, 1C).

Our findings indicate that under standard conditions of synthesis and water-solubilization with MAA, the CdSe QDs were not cytotoxic (Figure 1A). All TOPO-capped QDs were maintained in an inert atmosphere prior to alterations. A high concentration of MAA was added to TOPO-coated QDs to render them water-soluble. However if TOPO-coated QDs were initially subjected to air for 30 minutes and then modified with MAA, a dramatic dosedependent decrease in cellular viability was observed (from $98 \%$ to $21 \%$ at a QD concentration of $62.5 \mu \mathrm{g} / \mathrm{mL}$ ). Based on these results, we suspected that $\mathrm{O}_{2}$ in the air was oxidizing the surface of the CdSe QDs, leading to the observed cytotoxic effects.

To test this hypothesis, we used high-energy optical illumination to catalyze the oxidation process. Solutions of MAA-coated CdSe QDs were exposed to a UV-light source $\left(\lambda_{\mathrm{em}}=\right.$ $365 \mathrm{~nm}$ ) with a power density of $15 \mathrm{~mW} / \mathrm{cm}^{2}$ for 1 to $8 \mathrm{hrs}$ and then incubated with hepatocytes. Indeed, we observed a $6 \%, 42 \%, 83 \%$, and $97 \%$ decrease in viability when cells were incubated with CdSe QDs $(62.5 \mu \mathrm{g} / \mathrm{mL})$ after being exposed to UV-light for 1, 2, 4, and 8 hrs (Figure 1A).

Both air and UV light have been reported to induce and catalyze, respectively, the oxidation of nanoparticle surfaces ${ }^{24,26-28}$. After exposure to an oxidative environment, we observed a progressive change in the color and absorbance profile of the QD solution, a blue-shift in the excitonic fluorescence spectra, a broad red-shifted fluorescence peak adjacent to the excitonic fluorescence peak, and a decrease in the quantum yield (Figures 2A, 2B). These results are in agreement with previous oxidation studies ${ }^{26,27}$. Shifts in the absorbance and fluorescence spectra occur because of a decrease in the size of the nanoparticle (loss of surface atoms due to oxidation), while the broad red-shifted fluorescence peak can be attributed to the formation of lower-energy bandgaps induced by newly formed defect structures. It has been postulated that $\mathrm{O}_{2}$ molecules oxidize chalcogenide atoms ( $\mathrm{Se}, \mathrm{S}$ ) located on the surface of the QDs to form oxides $\left(\mathrm{SeO}_{2}, \mathrm{SO}_{4}{ }^{2-}\right)^{27,29}$. In the case of $\mathrm{CdSe}$ QDs, these $\mathrm{SeO}_{2}$ molecules desorb from the surface, leaving behind "dangling" reduced $\mathrm{Cd}$ atoms. Thus, prolonged exposure of QDs to an oxidative environment can cause the decomposition of the CdSe nanocrystal, thereby leading to desorption of Cd ions or $\mathrm{CdSe}$ complexes from the core QD. In the UV-light experiment, we also observed a marked increase in cytotoxicity with oxidation time, suggesting that free cadmium was progressively released from the QDs.

We theorized that release of free cadmium thereby led to cytotoxicity via conventional mechanisms of heavy metal toxicity. In order to lend credence to this hypothesis, we 
correlated the amount of free $\mathrm{Cd}^{2+}$ with the degree of observed hepatotoxicicty using inductively coupled plasma optical emission spectroscopy (ICP/OES). QD samples were processed to remove intact nanoparticles by acid precipitation and centrifugation, and the remaining solution was tested for free cadmium by ICP/OES. Since acid can slowly etch QDs, we first measured the $\mathrm{Cd}^{2+}$ concentration of non-oxidized MAA-QDs to determine a baseline $\mathrm{Cd}^{2+}$ concentration and to verify that the ICP/OES Cd${ }^{2+}$ measurements were not due to acid degradation of QDs. Relatively high levels of $\mathrm{Cd}^{2+}$ were found both in the airoxidized (126 ppm) and UV-exposed samples ( $82 \mathrm{ppm}$ ), while low levels were found in a solution of non-oxidized CdSe QDs (6 ppm). In our culture model, these $\mathrm{Cd}^{2+}$ ion concentrations correlated with high $(95-98 \%)$ and low $(0 \%)$ rates of cytotoxicity. This data correlates well with previous reports on rat hepatocyte cultures where exposure to 100-400 $\mu \mathrm{M}(11-44 \mathrm{ppm}) \mathrm{Cd}^{2+}$ ions led to significant cell death ${ }^{17}$. Some cell types, such as cardiomyocytes, are sensitive to even lower levels of cadmium exposure $(0.1 \mathrm{uM}$ or $11 \mathrm{ppb})$, while other cell types are more resistant ${ }^{18}$. Based on this data, it appears that surface oxidation of the CdSe nanocrystals during processing, via air-induced or UV-catalyzed oxidation, resulted in release of $\mathrm{Cd}$ surface atoms. The free cadmium in solution thereby rendered the QD solution cytotoxic via known mechanisms of cadmium hepatotoxicity (binding of thiol groups in the mitochondria) ${ }^{19}$. When the surface is not oxidized, however, the $\mathrm{Cd}$ atoms on the surface of the nanoparticles are bound to neighboring Se atoms and to the stabilizing ligand and are relatively innocuous.

These findings indicate that processing strategies, surface coatings, and use in biological applications that prevent surface oxidation should improve biocompatibility of CdSe-core QDs whereas oxidative environments should promote cytotoxicity. For cell-based studies using QDs, it is important to store and maintain them in an inert atmosphere $\left(\mathrm{N}_{2}\right)$ or with antioxidants, as our findings indicate that exposure to air prior to capping can render the QDs cytotoxic. Additionally, an important implication of our findings is for in vivo applications (e.g. tumor targeting and imaging). We have previously observed that QDs, like other particulates, are cleared from circulation of live mice by the macrophages of the reticuloendothelial system (RES) in the liver and the spleen ${ }^{11}$. Phagocytosis of nanoparticles would expose QDs to highly oxidative environments $\left(\mathrm{H}_{2} \mathrm{O}_{2}\right)$ intended for elimination of microorganisms. We therefore tested the $\mathrm{QD}$ response to $1 \mathrm{mM} \mathrm{H}_{2} \mathrm{O}_{2}$ for $24 \mathrm{~h}$ in vitro. Our results indicate that free cadmium $(24 \mathrm{ppm})$ is indeed released under these conditions in vitro (Figure 3B), leading to the possibility of QD toxicity in some in vivo applications. Strategies to protect the QD surface from highly oxidative environments or incorporation of surface coatings (such as polyethylene glycol) that promote escape from the RES may therefore be critical to the success of in vivo QD applications.

\section{Nanoparticle coatings to reduce surface oxidation}

Surface ligands have been shown to decrease the QD surface oxidation by limiting transport of oxygen to the surface as has been observed for self-assembled monolayers on gold films ${ }^{26}$. In a similar manner, we would expect the addition of surface coatings ( $\mathrm{ZnS}$, DHLA $^{8}$, BSA $^{30}$, polyacrylate ${ }^{9}$ ), typically added to increase the quantum yield and stability of nanoparticles, to decrease the surface oxidation and resulting cytotoxicity. 
To test the effects of surface coatings on cytotoxicity, CdSe QDs were capped with 1-2 monolayers of ZnS, yielding orange-emitting $\left(\lambda_{\mathrm{em} \max }=600 \mathrm{~nm}\right) \mathrm{ZnS} / \mathrm{CdSe}_{\mathrm{QDs}}{ }^{21,24}$. The presence of the capping layer was confirmed by an increased, red-shifted fluorescence peak and characteristic changes in the absorbance spectra. As with the uncapped nanoparticles (Figures 1-3), surface oxidation was studied by treatment with: (1) exposure of TOPOcapped QDs to air for up to 12 hours, followed by water solubilization with MAA, and (2) exposure to $365 \mathrm{~nm}$ UV light for up to 8 hours. QDs were assayed for cytotoxicity in primary hepatocyte cultures as described above.

Our findings indicate that $\mathrm{ZnS}$ capping virtually eliminated cytotoxicity due to oxidation by air during processing, which correlates well with previous XPS measurements showing that the addition of a $\mathrm{ZnS}$ capping layer renders QDs less susceptible to oxidation than the CdSe core alone ${ }^{24}$ (Figure 4A). Spectral data indicated that the CdSe core is intact after $12 \mathrm{~h}$ of oxidation in air similar to previous studies, though some disruption of the $\mathrm{ZnS}$ cap was observed as a blue-shift in the absorbance spectra (Figure 4B). ZnS capping, however, did not fully eliminate cytotoxicity induced by UV photooxidation (Figure 4A). ICP/OES data indicated high levels of free $\mathrm{Cd}$ in solution after $8 \mathrm{~h}$ of photooxidation, confirming that the $\mathrm{ZnS}$ cap alone does not fully protect the CdSe core under these conditions (Figure 4C). While $\mathrm{ZnS}$ is less susceptible to oxidation than CdSe, the loss of sulfur atoms to form sulfate ions is possible, leaving the CdSe core vulnerable to oxidation ${ }^{27,29}$. The reported stability of ZnS-capped CdSe QDs is likely due to the relatively slow oxidation by air under ambient, uncatalyzed conditions. In contrast, high-energy radiation catalyzes this oxidation process, leading to the photodecomposition of QDs.

Thus far, we have explored the influence of small organic ligands (MAA) on the photooxidative process of QDs and its affect on cytotoxicity. To explore the potential effects of large organic molecules on the cytotoxicity of QDs, we coated and cross-linked large organic-based molecules onto the surface of QDs. Bovine Serum Albumin (BSA) molecules were conjugated to the surface of MAA-coated ZnS-capped CdSe QDs with a water-based carbodiimide compound (EDAC) to produce a non-desorbable protein shell. Similar strategies have been used to coat the surface of QDs with dendrimer-like compounds ${ }^{31}$, glass $^{4}$, and amphiphilic polymers ${ }^{9}$. These types of QDs have an improved stability against flocculation, increased quantum yield, and surface ligands that allow further bioconjugation. As seen in Figure 5A, the addition of a BSA coat provided increased protection from photooxidation-mediated cytotoxicity over $\mathrm{ZnS}$ capping alone. Specifically, BSA-coated, ZnS-capped QDs were found to have reduced cytotoxicity at $0.25 \mathrm{mg} / \mathrm{mL}$ (98\% viability) as compared to ZnS-capped QDs (66\%) at the same concentration. ICP/OES measurements corroborated with these conclusions as measured by the reduction in release of free cadmium in BSA-coated dots (21 ppm) as compared to ZnS-capped QDs (36 ppm). It can be concluded that the protein-shell slows the oxidation process, likely by acting as a physical diffusion barrier for $\mathrm{O}_{2}$ molecules to the surface. Toxicity was not completely eliminated by BSA capping, however, as evidenced by substantial cell death (95\%) at the highest QD concentration tested $(1.0 \mathrm{mg} / \mathrm{mL})$ after $8 \mathrm{~h}$ of UV exposure. Under these conditions, spectral data also suggested significant surface oxidation (decrease in quantum confinement peak and $6 \mathrm{~nm}$ blue-shift in fluorescence peak) consistent with that observed for uncapped QDs (Figure 5B, 5C). 
To relate our toxicity results with surface coatings used by other groups, we also synthesized DHLA-capped ${ }^{8,32,33}$ and purchased polyacrylate/streptavidin-coated (Quantum Dot Corporation) ZnS/CdSe QDs. Exposure of these QDs to similar amounts of UV (8hrs at $15 \mathrm{~mW} / \mathrm{cm} 2$ ) also resulted in release of low levels of free cadmium, as measured by ICPOES (32 ppm for DHLA QDs and $24 \mathrm{ppm}$ for commercial QDs for $\sim 250 \mathrm{ug} / \mathrm{mL}$ samples). Based on the correlation between hepatocyte toxicity and free cadmium measured by ICP, we would surmise that these QD formulations would also be cytotoxic at $1 \mathrm{mg} / \mathrm{mL}$ after UV exposure. Thus, even with the high-performing commercially available QDs, release of free Cd over time is a concern.

In summary, the successive addition of an inorganic layer, small organic ligands, and a cross-linked organic shell progressively minimizes the cytotoxicity of QDs, increasing the biocompatibility of CdSe QDs. For typical in vitro cell-imaging studies, such high concentrations are not commonly used. A concentration of $1 \mathrm{mg} / \mathrm{mL}$ of QDs is approximately 10 fold higher than the concentrations used by Simon and co-workers for labeling HeLa cells ${ }^{8}$ and roughly 250 times higher than the concentrations used by Wu et al. for targeting of Her 2 epitopes on breast cancer cells ${ }^{9}$. Furthermore, for conventional longterm cell imaging studies, continuous excitation would not typically be employed. Therefore, our results indicate the upper concentration limit and light exposure at which QDs can be utilized for cell-imaging studies and that careful attention to the processing and coating details of QDs will minimize or prevent cellular toxicity.

For in vivo applications, however, protection of the CdSe surface from oxidation may be more critical. In two previous studies, relatively high concentrations of QDs $(\sim 100 \mathrm{ug} / \mathrm{mL}$ of $\mathrm{CdSe} / \mathrm{ZnS}$ ) were delivered to mice through intravenous injection ${ }^{11,13}$. Once delivered to the body systemically, there are three fates for circulating particles - clearance intact through the kidney, breakdown into smaller particles, or sequestering of the particles (where they are protected from short-term breakdown, but also prevented from clearing the body). Since QD conjugates are typically spherical, greater than $7 \mathrm{~nm}$ in diameter, and negatively charged, clearance through the kidney glomerulus is likely to be a rare event ${ }^{34}(<0.1 \%$ clearance based on studies with albumin ${ }^{35}$ ) unless they are first broken down into smaller particles. Our experiments suggest that, even with multiple inorganic/organic surface coatings, oxidation of the particles can occur, releasing free cadmium. While extreme conditions were used to generate the acute cytotoxicity observed, slower release may result in chronic effects of cadmium exposure, such as kidney damage ${ }^{36}$. Penetration of UV light through the skin $^{37}$ and/or oxidation due to inflammatory responses could induce cadmium release via surface oxidation; therefore, highly protective surface coatings would be critical to mitigating the toxicity of CdSe QDs in vivo. BSA and polyacrylate coatings are unlikely to be the only surface coatings that substantially reduce surface oxidation. In fact, we expect that other coating strategies currently employed or under development (i.e. polymer coatings, lipidmicelles with protein cross-linking) would offer varying degrees of protection from surface oxidation and resultant cytotoxicity. The data presented in this study should provide design criteria to quantitatively evaluate the robustness of a proposed coating strategy for in vitro or in vivo applications. Although other cadmium-containing QDs exist (CdS, CdTe), CdSe has proven to be the most utilized QD core material for biological experiments, likely due to a visible fluorescence range (blue to near infrared) and well-investigated synthesis methods. 
Though the other constituent elements of these QDs ( $\mathrm{Zn}, \mathrm{Se}, \mathrm{S})$ can be toxic in high doses, the concentrations exposed to the hepatocytes in these experiments are below cytotoxic levels $(<4 \mathrm{mM})^{38}$. Furthermore, the addition of either $\mathrm{Zn}$ or Se (both dietary supplements) has been shown to reduce, rather than exacerbate, cadmium toxicity ${ }^{39}$.

\section{Probing effects of QD on higher-order cellular functions: Application to live cell labeling}

Thus far, our experiments have demonstrated that $\mathrm{ZnS} / \mathrm{CdSe}$ QDs are not cytotoxic to primary hepatocytes at low concentrations $(<0.25 \mathrm{mg} / \mathrm{mL})$ and relatively short periods of UV exposure ( $\leq 1$ hour). Working within these parameters, we sought to demonstrate the utility of QDs as a tool for long-term, live cell labeling in a sensitive model system of liver tissue, the site of cadmium toxicity in vivo. Indeed, long-term, live cell labeling is one area where QDs show potential benefits over both conventional fluorescent dyes (e.g. calcein) and fluorescent proteins (e.g.. green fluorescent protein). Rather than using viability as a crude measure of toxicity, we investigated whether the migration or differentiated function of rat hepatocytes (a fragile, primary cell type) would be altered by labeling with $\mathrm{ZnS}$ capped CdSe QDs. In order to create a model of liver tissue that is stably differentiated over many days, we exploited a hepatocyte culture model we have previously developed ${ }^{40}$. Specifically, hepatocytes are co-cultivated with non-parenchymal cells that promote differentiated function of hepatocytes in vitro. Furthermore, in order to track hepatocyte migration, we formed organized cellular arrays using a micropatterning technique described in detail elsewhere ${ }^{41}$. Red-emitting CdSe QDs were capped with ZnS, coated with polyethylene glycol to prevent non-specific binding, and conjugated to a hepatocyte ligand, epidermal growth factor (EGF). Hepatocytes were then labeled by endocytosis of EGFconjugated QDs $\left(\lambda_{\mathrm{em} \max }=630 \mathrm{~nm}\right)$ and micropatterned in arrays surrounded by fibroblasts.

On day 1 of co-culture, organized colonies of hepatocytes were identified morphologically under phase contrast microscopy and observed to correlate with red QD fluorescence (Figures 6A, 6B). Over 7 days in culture, hepatocytes reorganized due to cell migration. Again, phase contrast microscopy allowed correlation of hepatocyte morphology (polygonal cells with distinct nuclei) with QD fluorescence (Figures 6C, 6D). These data indicate the potential to label live cells for one week in culture. In addition to morphologic evidence of cell viability and microscopic evidence of cell migration, liver-specific function of these fragile, primary cells was assayed by measurement of daily albumin production. Our data indicate no deleterious effects of QD-labeling on hepatocellular function (Figure 6E) when compared to unlabeled controls. Thus, organically-coated, ZnS-capped CdSe QDs were found to be 'biocompatible' with hepatic tissue as determined by cell viability, migration, and differentiated function over two weeks in culture. In the future, QDs could be utilized in a similar fashion to track a number of dynamic cellular processes including stem cell progeny, cancer metastases, morphogenesis, and wound healing.

\section{Summary and Conclusions}

In summary, we have shown that $\mathrm{CdSe}$-core quantum dots are indeed cytotoxic under certain conditions. Specifically, surface oxidation through a variety of pathways led to the formation 
of reduced $\mathrm{Cd}$ on the QD surface and release of free cadmium ions, and correlated with cell death. Surface coatings such as $\mathrm{ZnS}$ and BSA were shown to significantly reduce, but not eliminate cytotoxicity. While many groups make use of a $\mathrm{ZnS}$ capping layer, synthesis methods using $\mathrm{CdO}$ as a precursor allow production of highly luminescent $\mathrm{CdSe}$ nanocrystals that do not require an inorganic capping layer ${ }^{22,42}$. Additionally, others are interested in minimizing the size of the nanoparticle shell and have made use of uncapped CdSe or CdS QDs for biological labeling experiments ${ }^{43,44}$. For these QDs, an organic capping layer must bear the burden of preventing surface oxidation, and therefore cytotoxicity.

Nonetheless, coated QDs were shown to be useful for long-term live cell labeling of hepatic tissue in vitro without deleterious effects on viability, migration, or differentiated function. The use of QDs in vivo, however, must be critically examined, as our results suggest $\mathrm{Cd}$ release is a possibility over time. In comparison to other nanomaterials that may exhibit novel mechanisms of toxicity due to their size (e.g. inhalation of carbon nanotubes), CdSe QDs were found to induce cell death due to their inherent chemical composition. Rather than signal an end to the future of QD labeling, these findings serve to suggest parameters for synthesis, processing, and surface coating to minimize heavy metal toxicity in biological applications and establish a quantitative framework to evaluate the potential of this and other types of nanomaterials (e.g., InP, InAs, Ag nanoparticles).

\section{Supplementary Material}

Refer to Web version on PubMed Central for supplementary material.

\section{Acknowledgments}

We thank Elliot Hui for assistance with micropatterning, Jennifer Felix for hepatocyte isolation, and Mike Sailor for providing facilities for QD synthesis. Funding provided by DARPA-MTO, David and Lucile Packard Foundation, NIH T32 HL07089, NIH NDDK, University of Toronto (WC), and the Connaught Foundation (WC).

\section{References}

1. Lam CW, James JT, McCluskey R, Hunter RL. Toxicol Sci. 2003

2. Warheit DB, Laurence BR, Reed KL, Roach DH, Reynolds GA, Webb TR. Toxicol Sci. 2003

3. Chan WC, Nie S. Science. 1998; 281:2016-2018. [PubMed: 9748158]

4. Bruchez M, Moronne M, Gin P, Weiss S, Alivisatos AP. Science. 1998; 281:2013-2016. [PubMed: 9748157]

5. Chan WC, Maxwell DJ, Gao X, Bailey RE, Han M, Nie S. Curr Opin Biotechnol. 2002; 13:40-46. [PubMed: 11849956]

6. Parak WJ, Boudreau R, Le Gros M, Gerion D, Zanchet D, Micheel CM, Williams SC, Alivisatos AP, Larabell C. Adv. Mater. 2002; 14:882-885.

7. Hanaki K, Momo A, Oku T, Komoto A, Maenosono S, Yamaguchi Y, Yamamoto K. Biochem Biophys Res Commun. 2003; 302:496-501. [PubMed: 12615061]

8. Jaiswal JK, Mattoussi H, Mauro JM, Simon SM. Nat Biotechnol. 2003; 21:47-51. [PubMed: 12459736]

9. Wu X, Liu H, Liu J, Haley KN, Treadway JA, Larson JP, Ge N, Peale F, Bruchez MP. Nat Biotechnol. 2003; 21:41-46. [PubMed: 12459735]

10. Dahan M, Levi S, Luccardini C, Rostaing P, Riveau B, Triller A. Science. 2003; 302:442-445. [PubMed: 14564008] 
11. Akerman ME, Chan WC, Laakkonen P, Bhatia SN, Ruoslahti E. Proc Natl Acad Sci USA. 2002; 99:12617-12621. [PubMed: 12235356]

12. Dubertret B, Skourides P, Norris DJ, Noireaux V, Brivanlou AH, Libchaber A. Science. 2002; 298:1759-1762. [PubMed: 12459582]

13. Larson DR, Zipfel WR, Williams RM, Clark SW, Bruchez MP, Wise FW, Webb WW. Science. 2003; 300:1434-1436. [PubMed: 12775841]

14. Seydel C. Science. 2003; 300:80-81. [PubMed: 12677056]

15. Dagani R. Chemical and Engineering News. 2003; 81:30-33.

16. Colvin VL. Nat Biotechnol. 2003; 21:1166-1170. [PubMed: 14520401]

17. Santone KS, Acosta D, Bruckner JV. J Toxicol Environ Health. 1982; 10:169-177. [PubMed: 7131585]

18. Limaye DA, Shaikh ZA. Toxicol Appl Pharmacol. 1999; 154:59-66. [PubMed: 9882592]

19. Rikans LE, Yamano T. J Biochem Mol Toxicol. 2000; 14:110-117. [PubMed: 10630425]

20. Liu J, Kershaw WC, Klaassen CD. In Vitro Cell Dev Biol. 1990; 26:75-79. [PubMed: 2307641]

21. Hines MA, Guyot-Sionnest P. J. Phys. Chem. 1996; 100:468-471.

22. Qu LH, Peng ZA, Peng XG. Nano Letters. 2001; 1:333-337.

23. Peng XG, Schlamp MC, Kadavanich AV, Alivisatos AP. J. AmChem. Soc. 1997; 119:7019-7029.

24. Dabbousi BO, Rodriguezviejo J, Mikulec FV, Heine JR, Mattoussi H, Ober R, Jensen KF, Bawendi MG. J. Phys. Chem. B. 1997; 101:9463-9475.

25. Murray CB, Norris DJ, Bawendi MG. J. AmChem. Soc. 1993; 115:8706-8715.

26. Aldana J, Wang YA, Peng XG. J. AmChem. Soc. 2001; 123:8844-8850.

27. Spanhel L, Haase M, Weller H, Henglein A. J Am Chem Soc. 1987; 109:5649-5655.

28. Katari JEB, Colvin VL, Alivisatos AP. J. Phys. Chem. 1994; 98:4109-4117.

29. Alivisatos AP. J. Phys. Chem. 1996; 100:13226-13239.

30. Gao X, Chan WC, Nie S. J Biomed Opt. 2002; 7:532-537. [PubMed: 12421118]

31. Wang YA, Li JJ, Chen HY, Peng XG. J. AmChem. Soc. 2002; 124:2293-2298.

32. Mattoussi H, Mauro JM, Goldman ER, Anderson GP, Sundar VC, Mikulec FV, Bawendi MG. Journal of the American Chemical Society. 2000; 122:12142-12150.

33. Medintz IL, Clapp AR, Mattoussi H, Goldman ER, Fisher B, Mauro JM. Nature Materials. 2003; 2:630-638. [PubMed: 12942071]

34. Rose, BD., Rennke, HG. Renal Pathophysiology. Williams \& Wilkins: Baltimore; 1994.

35. Lund U, Rippe A, Venturoli D, Tenstad O, Grubb A, Rippe B. Am J Physiol Renal Physiol. 2003; 284:F1226-1234. [PubMed: 12620929]

36. Brzoska MM, Kaminski M, Supernak-Bobko D, Zwierz K, Moniuszko-Jakoniuk J. Arch Toxicol. 2003; 77:344-352. [PubMed: 12799774]

37. Elisseeff J, Anseth K, Sims D, McIntosh W, Randolph M, Langer R. Proc Natl Acad Sci USA. 1999; 96:3104-3107. [PubMed: 10077644]

38. Stacey NH, Klaassen CD. J Toxicol Environ Health. 1981; 7:139-147. [PubMed: 6455537]

39. Goyer RA. Am J Clin Nutr. 1995; 61:646S-650S. [PubMed: 7879732]

40. Bhatia SN, Balis UJ, Yarmush ML, Toner M. Faseb J. 1999; 13:1883-1900. [PubMed: 10544172]

41. Bhatia SN, Yarmush ML, Toner M. J Biomed Mater Res. 1997; 34:189-199. [PubMed: 9029299]

42. Qu LH, Peng XG. Journal of the American Chemical Society. 2002; 124:2049-2055. [PubMed: 11866620]

43. Winter JO, Liu TY, Korgel BA, Schmidt CE. Advanced Materials. 2001; 13:1673-1677.

44. Kloepfer JA, Mielke RE, Wong MS, Nealson KH, Stucky G, Nadeau JL. Appl. Environ. Microbiol. 2003; 69:4205-4213. [PubMed: 12839801] 
$A$
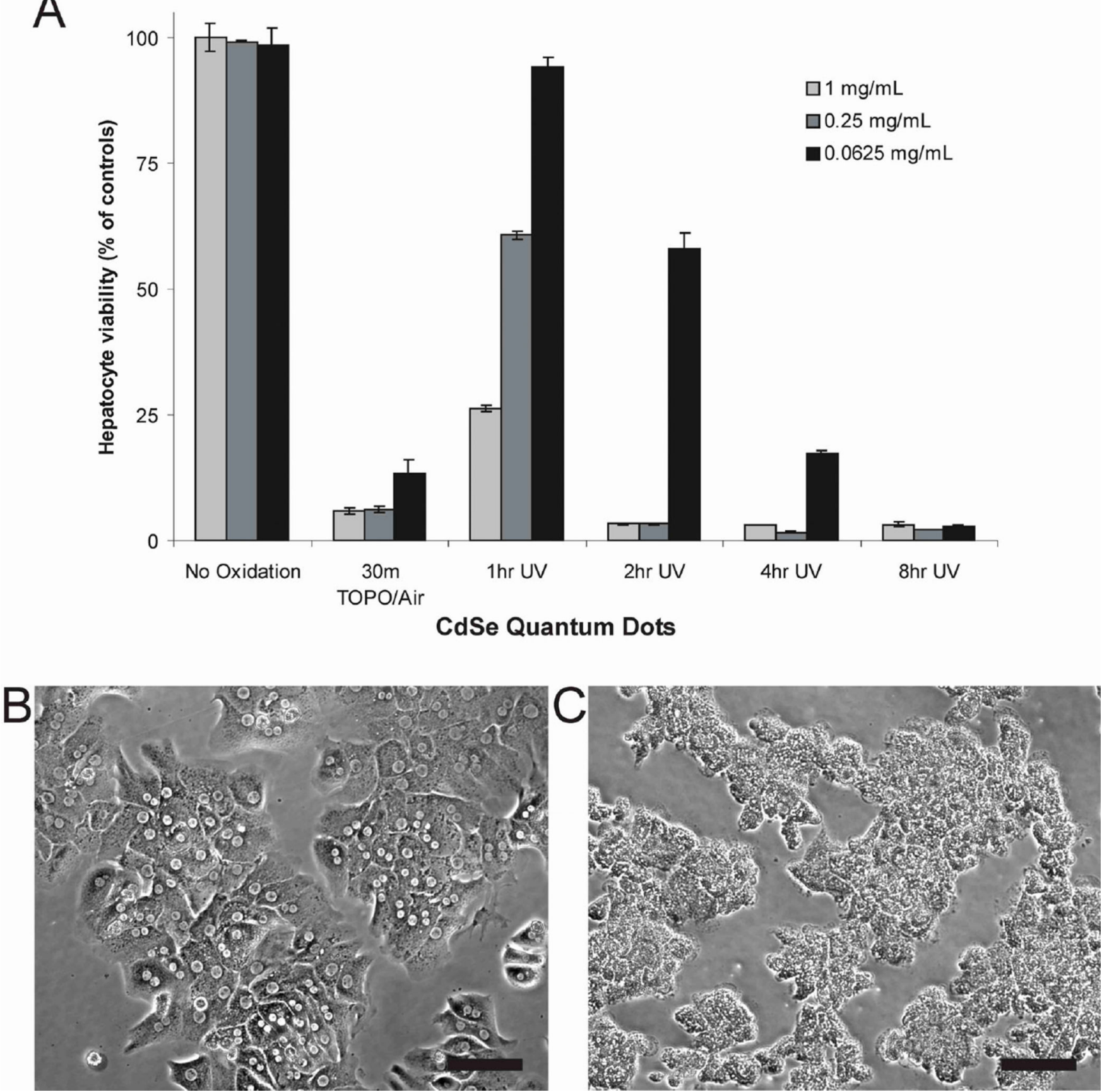

Figure 1. Toxicity Of CdSe Quantum Dots In Liver Culture Model Is Dependent On Processing Conditions And Nanoparticle Dose

(A) Hepatocyte viability as assessed by mitochondrial activity of QD-treated cultures relative to untreated controls. Thirty minutes of exposure to air while TOPO-capped renders QDs highly toxic at all concentrations tested. Ultraviolet light exposure also induces toxicity that increases with exposure time and is QD concentration-dependent. Biochemical assays of viability were confirmed via phase contrast microscopy where control hepatocyte cultures exhibited distinct intercellular boundaries, well-defined nuclei, and polygonal morphology (B). Nonviable cultures ( $<5 \%$ of controls) exposed to cytotoxic QDs exhibited granular 
cytoplasm, indistinct intercellular boundaries, undefined nuclei and evidence of blebbing (C). Scale bar corresponds to $100 \mu \mathrm{m}$. 


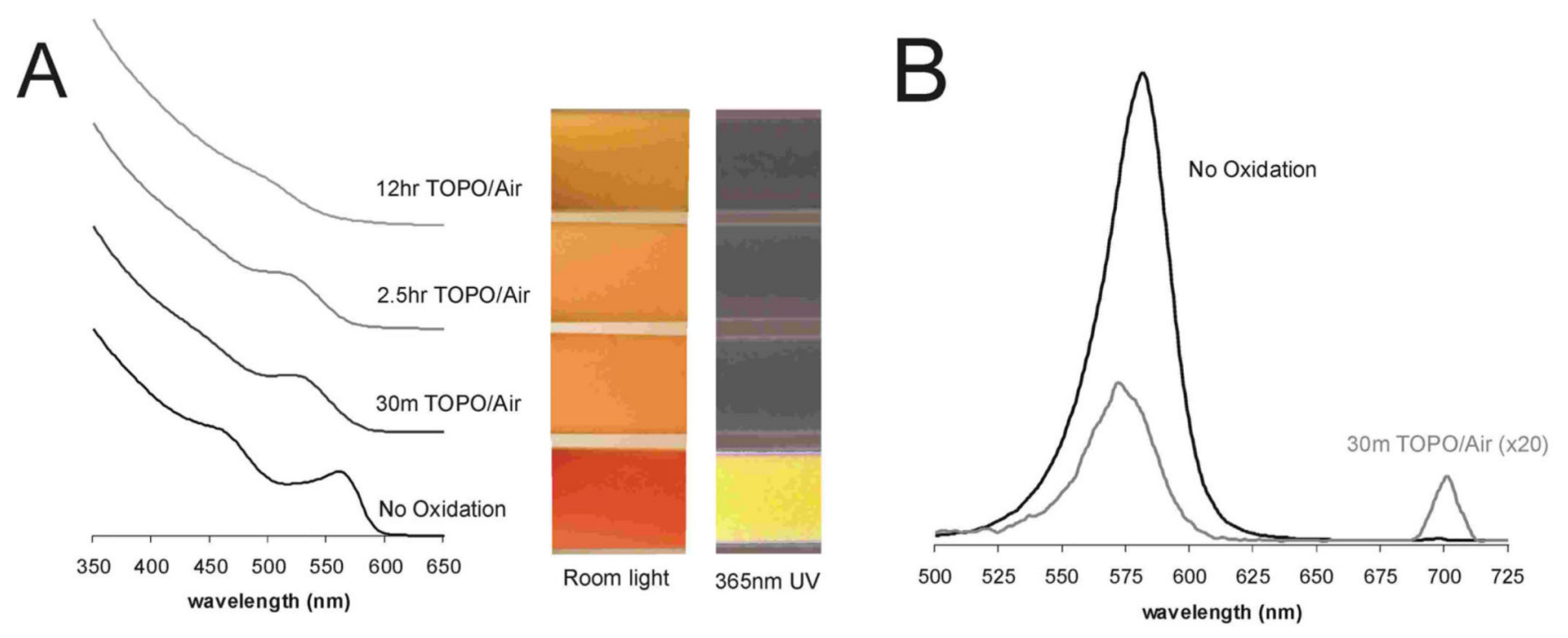

Figure 2. Toxicity Of QDs Correlates with Surface Oxidation, Decrease of QD size, and Disruption of Crystal Lattice

(A) Increased exposure to air of TOPO-capped QDs correlates with surface oxidation as indicated by a blue-shift in the first quantum confinement peak and decrease in peak amplitude of absorbance spectra (in chloroform). Observable changes in color of QD solutions due to changes in absorbance spectra (white light - shift from red/orange to yellow) and fluorescence spectra (UV light - loss of fluorescence) are also consistent with surface oxidation. (B) Exposure to air of TOPO-capped QDs produces changes in fluorescence spectra (blue-shift of fluorescence peak by $\sim 10 \mathrm{~nm}$ after $30 \mathrm{~min}$ ) consistent with a decrease in QD size due to removal of surface atoms. For comparison, the amplitude of 30min TOPO curve has been increased 20 fold to compensate for the loss in quantum yield. 
A CaSe QDs
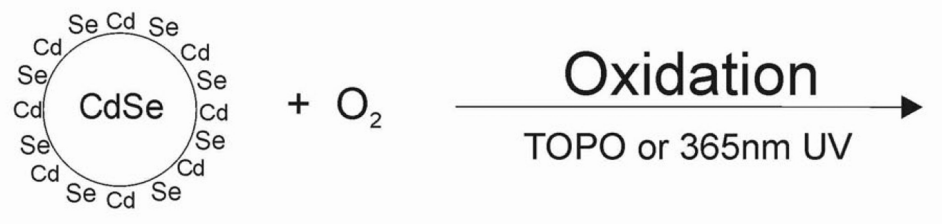

Removal of surface atoms
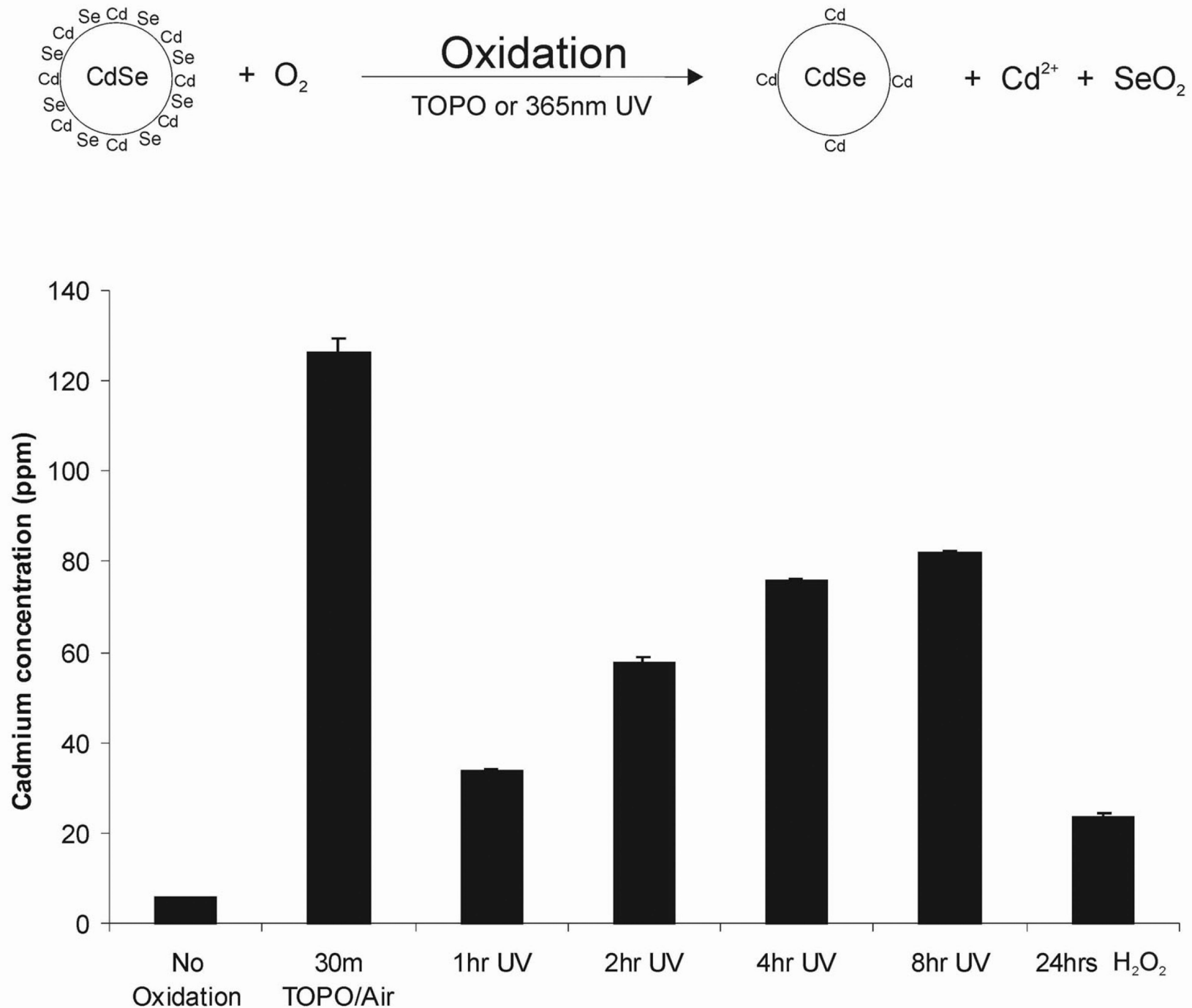

\section{CdSe QDs}

Figure 3. Surface Oxidation Leads to Release of Cadmium Ions

(A) Proposed mechanism of Cd release from the QD surface via either TOPO-mediated or UV-catalyzed surface oxidation. (B) Inductively coupled plasma optical emission spectroscopy (ICP/OES) measurements of free cadmium in $0.25 \mathrm{mg} / \mathrm{mL}$ solutions of QDs, indicating higher levels of free cadmium in all oxidized samples and increasing Cd levels with UV exposure time, correlating with cytotoxicity observed in Figure 1A. 

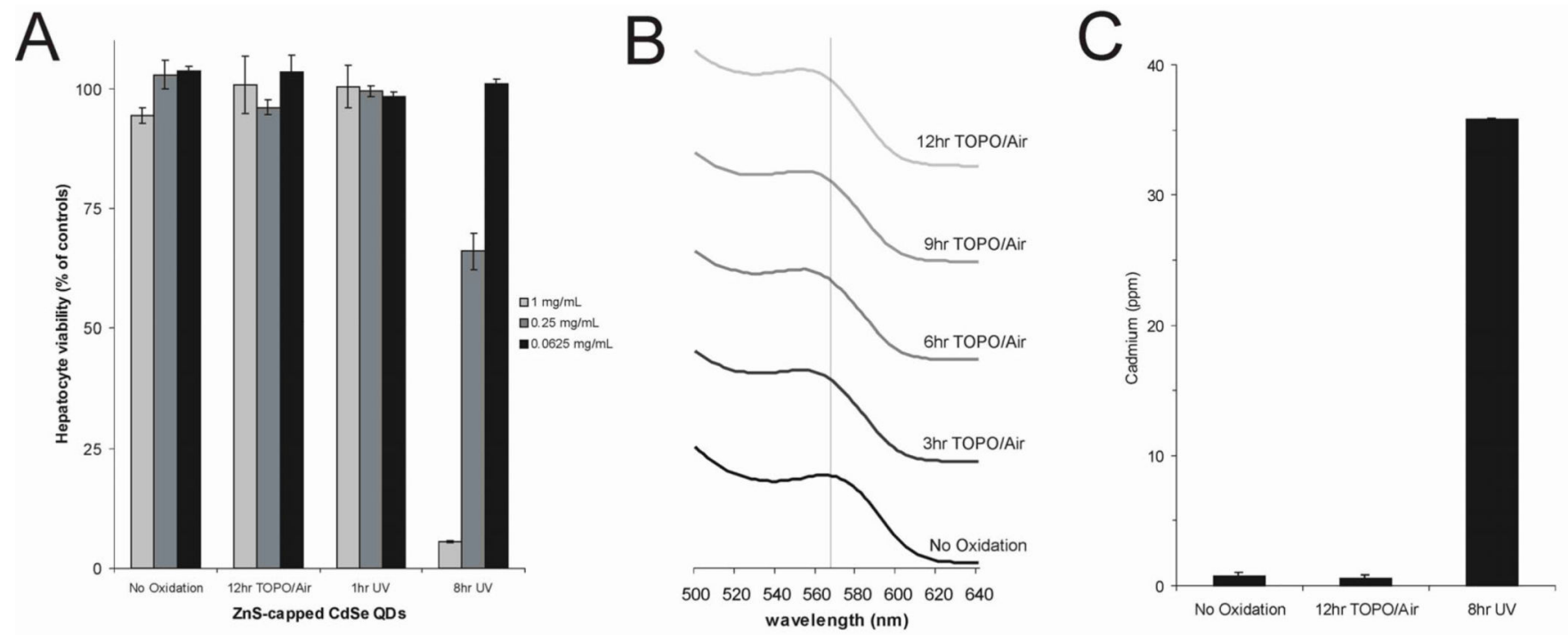

Figure 4. Effects of ZnS Surface Coating on Surface Oxidation, Release of Cadmium, and Cytotoxicity

(A) ZnS capping of CdSe QDs eliminates TOPO/air-induced cytotoxicity and reduces photooxidation-mediated cytotoxicity as indicated by viability of QD-treated hepatocytes compared to Figure 1A. (B) While the CdSe core remains intact, the absorbance spectra of TOPO/air treated QDs displays a blue shift, possibly indicating a disruption of the $\mathrm{ZnS}$ cap (in chloroform). (C) Free cadmium levels measured by ICP/OES in $0.25 \mathrm{mg} / \mathrm{mL}$ QD solution correlate with patterns of cytotoxicity observed in (A). 

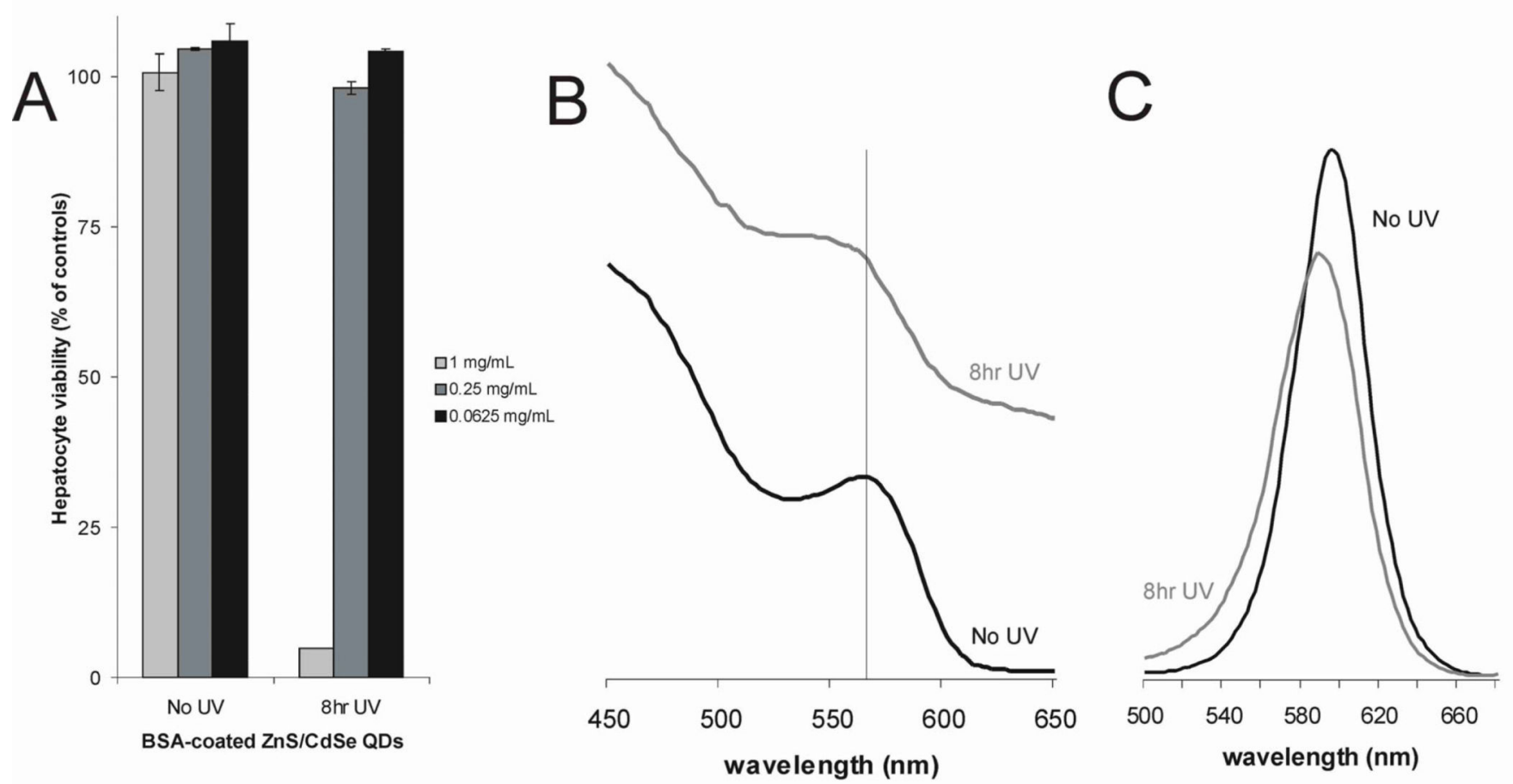

Figure 5. Effects of Bovine Serum Albumin Surface Coating on Surface Oxidation and Cytotoxicity

BSA-coated ZnS-capped QDs were photoxidized with ultraviolet light and compared to unexposed controls. (A) Photooxidation of BSA-coated QDs rendered QDs toxic only at high doses $(1 \mathrm{mg} / \mathrm{mL})$ after extensive exposure $(8 \mathrm{~h})$. Note the reduced cytotoxicity of 0.25 $\mathrm{mg} / \mathrm{mL}$ after $8 \mathrm{~h}$ of UV exposure (98\% viability) as compared to ZnS capped dots under similar conditions (66\% viability). (B) Photooxidation of BSA-coated QDs resulted in an observable change in the absorbance spectra (a decrease in the first quantum confinement peak), and (C) a change in the fluorescence spectra (a decrease in amplitude and blue-shift), corresponding to oxidation of the CdSe QD core. 

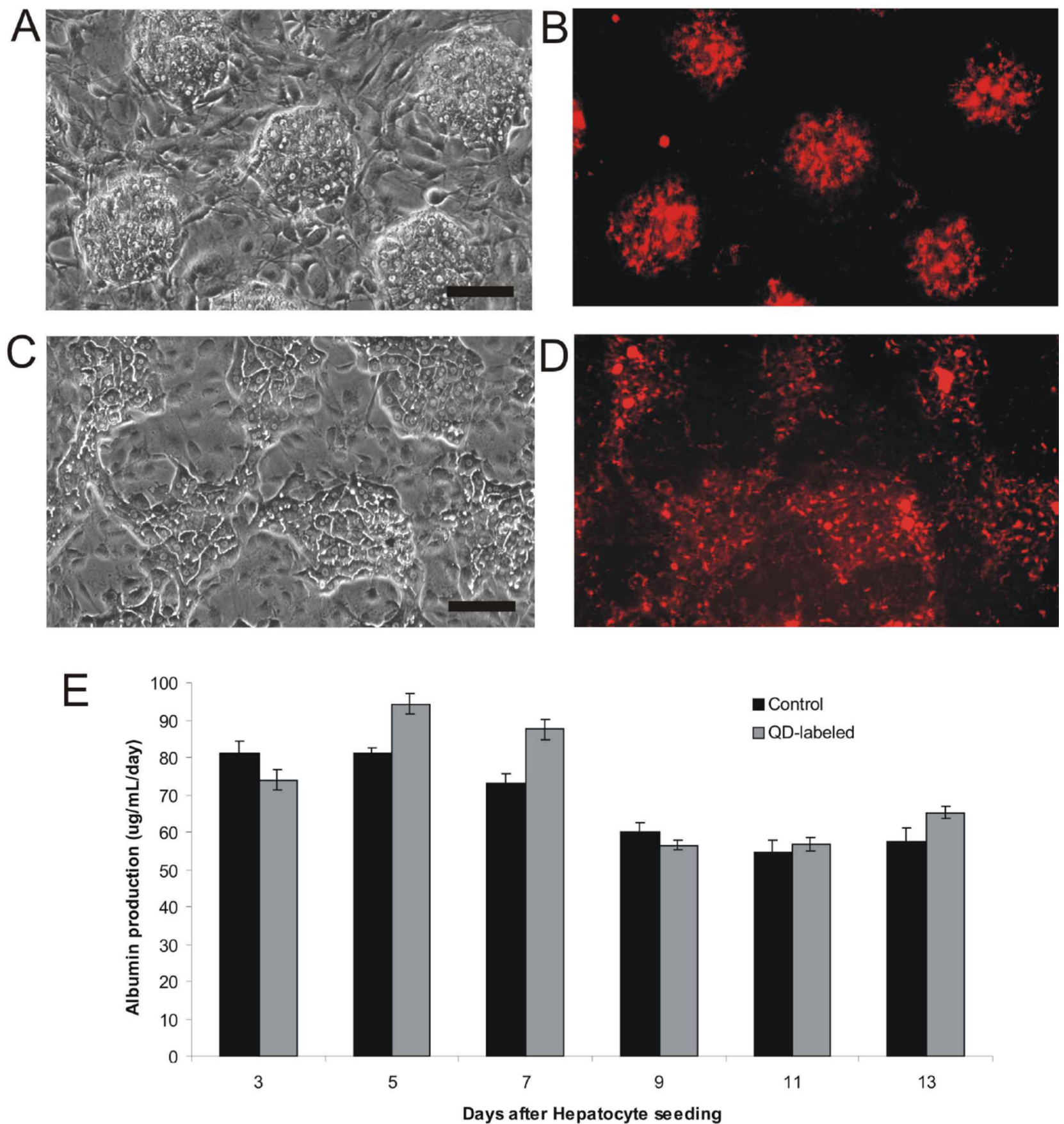

- Control

$\square$ QD-labeled

Figure 6. Application of Coated QDs to Long-term Tracking of Primary Cells Without Compromising Liver-specific Function

Hepatocytes were co-cultivated with non-parenchymal cells (3T3 fibroblasts) to support liver-specific functions in vitro ${ }^{40}$. Hepatocytes were labeled by endocytosis of EGF-coated red QDs. Co-cultures were organized in regular arrays using previously reported 'micropatterning' techniques ${ }^{41}$ and cell migration was monitored by phase contrast and fluorescence microscopy. (A) Phase contrast micrograph of micropatterned array of hepatocyte colonies $(\sim 100 \mu \mathrm{m})$ surrounded by fibroblasts on day 1 of co-culture and corresponding fluorescence image (B) of QD-labeled hepatocytes. Scale bar corresponds to 
$100 \mu \mathrm{m}(\mathrm{C})$ Phase contrast micrograph of co-culture demonstrates visible reorganization of hepatocyte colonies and corresponding fluorescence image (D) of QD-labeled hepatocytes after 7 days of co-culture. (E) Liver-specific functions of QD-labeled hepatocytes were comparable to control co-cultures for two weeks of culture as assessed by daily albumin secretion and averaged over two day periods. 